\section{JTI}

JOURNAL OF

TRAUMA AND INJURY

Received: October 13, 2020

Revised: December 3, 2020

Accepted: January 4, 2021

\section{Correspondence to}

II Jae Wang, M.D., Ph.D.

Department of Emergency Medicine, Pusan National University Hospital, Pusan

National University School of Medicine and Biomedical Research Institute, 179

Gudeok-ro, Seo-gu, Busan 49241, Korea

Tel: +82-51-240-7503

Fax: +82-51-253-6472

E-mail:jrmr9933@gmail.com

ORCID: https://orcid.org/0000-0003-

2479-2359

\title{
Chest Wall Reconstruction for the Treatment of Lung Herniation and Respiratory Failure 1 Month after Emergency Thoracotomy in a Patient with Traumatic Flail Chest
}

\author{
Junepill Seok, M.D. ${ }^{1}$, Il Jae Wang, M.D., Ph.D. ${ }^{2}$ \\ ${ }^{1}$ Department of Cardiovascular and Thoracic Surgery, Chungbuk National University \\ Hospital, Cheongju, Korea \\ ${ }^{2}$ Department of Emergency Medicine, Pusan National University Hospital, Pusan National \\ University School of Medicine and Biomedical Research Institute, Busan, Korea
}

We report a case of delayed chest wall reconstruction after thoracotomy. A 53-yearold female, a victim of a motor vehicle accident, presented with bilateral multiple rib fractures with flail motion and multiple extrathoracic injuries. Whole-body computed tomography revealed multiple fractures of the bilateral ribs, clavicle, and scapula, and bilateral hemopneumothorax with severe lung contusions. Active hemorrhage was also found in the anterior pelvis, which was treated by angioembolization. The patient was transferred to the surgical intensive care unit for follow-up. We planned to perform surgical stabilization of rib fractures (SSRF) because her lung condition did not seem favorable for general anesthesia. Within a few hours, however, massive hemorrhage (presumably due to coagulopathy) drained through the thoracic drainage catheter. We performed an exploratory thoracotomy in the operating room. We initially planned to perform exploratory thoracotomy and "on the way out" SSRF. In the operating room, the hemorrhage was controlled; however, her condition deteriorated and SSRF could not be completed. SSRF was completed after about a month owing to other medical conditions, and the patient was weaned successfully.

Keywords: Flail chest; Hyperbilirubinemia; Respiratory insufficiency; Thoracotomy 


\section{INTRODUCTION}

The principles of the treatment of severely dislocated rib fractures, including flail chest, have yet to be fully established, although "on the way out" or "thoracotomy for another indication" has been reported as a valid indication for rib fracture repair. In this article, we present a case of flail chest caused by blunt chest trauma in a patient who underwent rib fixation and chest wall reconstruction 1 month after an initial emergency thoracotomy.

\section{CASE REPORT}

We report the case of a 53-year-old female patient who was a pedestrian road traffic accident victim. The initial physical examination showed an open mandibular fracture and visible flail chest on the left lateral chest wall. The patient was intubated immediately on admission owing to dyspneic respiration. Whole-body computed tomography (CT) revealed multiple fractures of the bilateral ribs, clavicle, and scapula, and bilateral hemopneumothorax with severe lung contusions (Fig. 1). Active hemorrhage was also found in the anterior pelvis. After angioembolization of the pelvis following bilateral thoracostomies with 28 Fr chest tubes, the patient was transferred to the surgical intensive care unit (SICU) for follow-up. We planned to perform surgical stabilization of rib fractures (SSRF) because her lung condition did not seem favorable for general anesthesia. Within a few hours, however, massive hemorrhage ( $>400 \mathrm{~mL} /$ hour), presumably due to coagu- lopathy, drained through the thoracic drainage catheter. We performed an exploratory thoracotomy in the operating room.

A left-side, posterolateral thoracotomy incision was made on the damaged chest wall, and the 5th intercostal space was widely opened. Multiple sutures and ligations were performed for a $15-\mathrm{cm}$ lung laceration on the left lower lobe and multiple sites of active bleeding from intercostal vessels. However, the patient's hypoxia deteriorated owing to the severe lung contusions, even with maximum oxygen supplementation. After a discussion with anesthesiologists, we decided to stop the operation and transferred the patient to the SICU without definitive stabilization of the flail chest.

The patient underwent bed-side tracheostomy the following day, and her lung condition recovered slowly. Follow-up chest CT, which was taken 12 days later because of the patient's general condition, revealed further dislocated and collapsed rib fractures and herniation of the left lung due to the thoracotomy compared to the findings of the original chest CT (Figs. 1, 2). However, SSRF was further delayed owing to newly developed conjugated hyperbilirubinemia (direct bilirubin $>8 \mathrm{mg} / \mathrm{dL}$ ). The normalization of laboratory results took nearly a month. On hospital day 28, we were able to perform SSRF. After adhesiolysis of the herniated lung and ribs, a $15 \times 15 \mathrm{~cm}$ flail segment without a blood supply was completely separated from the chest wall. We returned the segment to its original place like a puzzle piece, stabilized it with titanium reconstruction plates and screws, and secured the trapezius muscle flap with Vicryl 3-0 continuous suture (Fig. 3A).
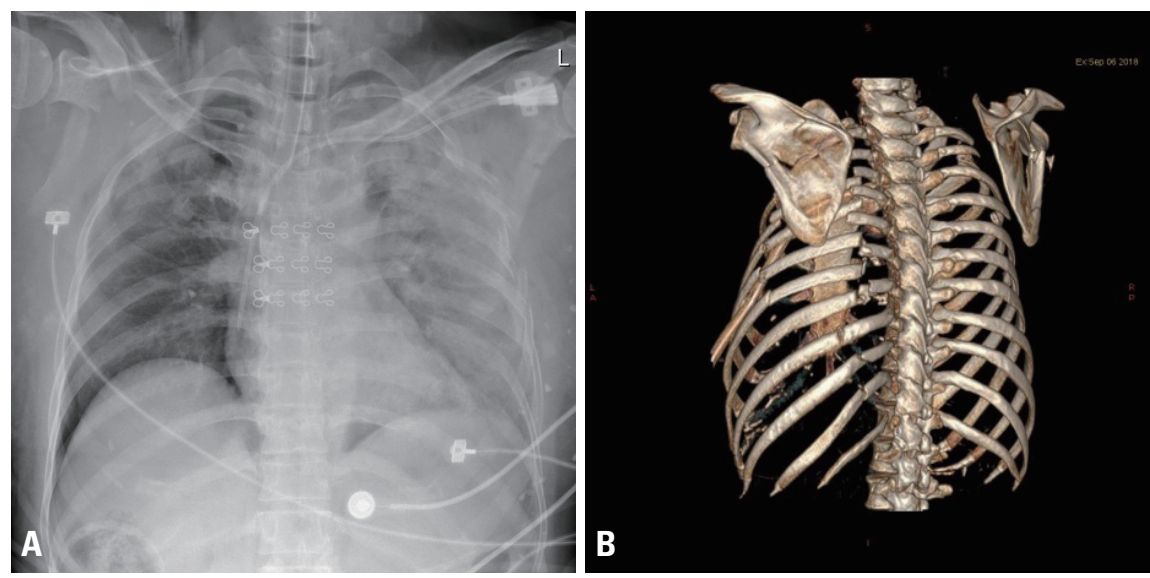

Fig. 1. Imaging on admission. (A) Initial chest radiograph. (B) Posterior view of three-dimensional computed tomography of the chest. 


\section{JTI}

On hospital day 34, the patient underwent open reduction and fixation for fractures of the mandible, clavicle, and scapula and was weaned from the mechanical ventilator. The tracheostomy was removed on hospital day 38 (Fig. 3B). The patient is currently receiving rehabilitation therapy.

\section{DISCUSSION}

Flail chest is a serious consequence of various thoracic injuries [1] and presents with at least two fractures of at least three consecutive ribs. These fractures are generally caused by injuries to the anterior or lateral thorax, or by sternal fractures and decompositions of multiple costochondral joints.
The treatment of flail chest is controversial. The conventional treatment of flail chest includes analgesia and respiratory support $[2,3]$. However, several prospective randomized controlled studies have revealed that surgical treatment of flail chest might lower the incidence of pneumonia, reduce medical costs, and shorten the lengths of ICU stay and mechanical ventilation compared to the conventional treatment [4-6].

"On the way out" or "thoracotomy for another indication" has been reported as a valid indication for rib fracture repair [2,7-10], because intercostal retraction during thoracotomy may cause further dislocation of ribs in the setting of rib fractures, as in our case. We initially planned to perform rib fixation immediately following acute hemorrhage control. As noted, fixation could not be completed owing to the patient's rapidly deteriorating
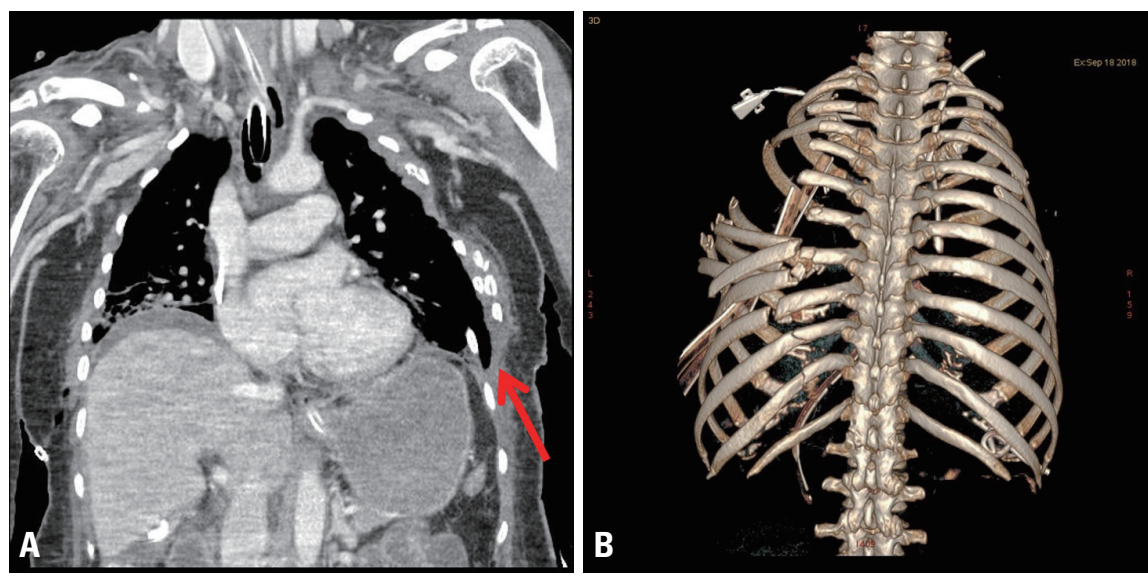

Fig. 2. Follow-up chest computed tomography on hospital day 12. (A) Herniated lung (arrow) between the broken ribs. (B) Posterior view of the collapsed chest wall.
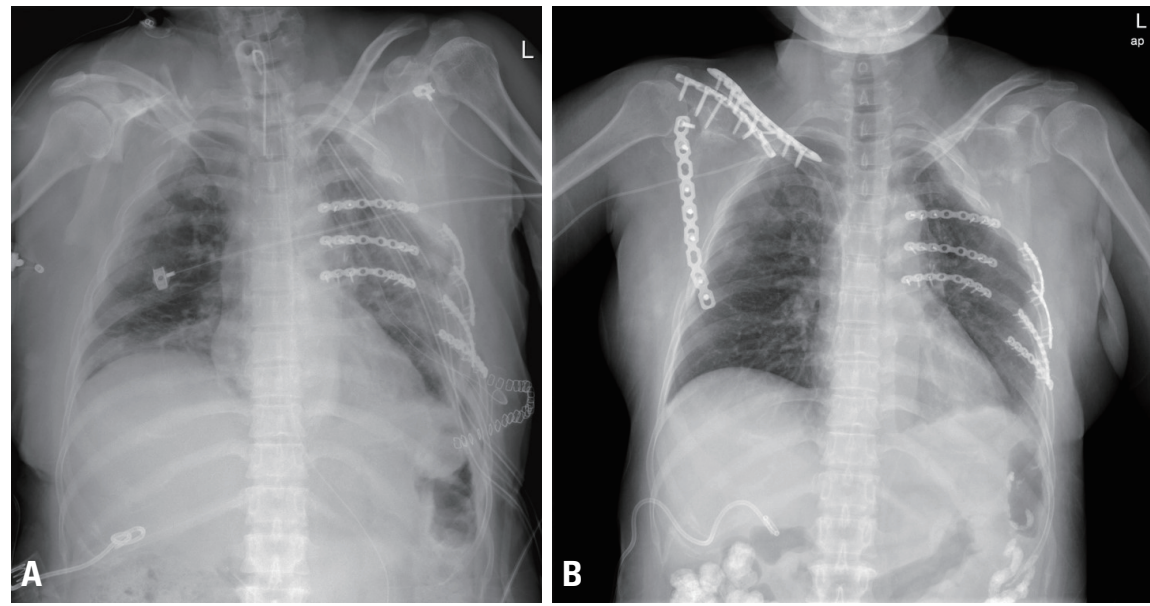

Fig. 3. Imaging after final reconstruction. (A) Immediate postoperative chest radiograph showing stabilized ribs with titanium reconstruction plates and screws. (B) Follow-up chest radiograph after removal of the tracheostomy tube. 
condition during the operation. As a result, the patient was dependent on mechanical ventilation for approximately a month owing to respiratory failure caused by flail motion of the chest wall. Further, a huge chest wall defect, including the herniated lung, caused profound oxygen saturation fluctuations during positional changes in the SICU. After stabilization of the flail chest, it took only 6 days to wean the patient from mechanical ventilation.

Conjugated hyperbilirubinemia following severe trauma without hepatic dysfunction is an uncommon situation. In a case report published in 2009 [11], the authors reported that patients with severe (especially abdominal or pelvic) trauma may develop a typical pattern of conjugated hyperbilirubinemia owing to massive bilirubin overload, severe shock, and cholestatic intrahepatic dysfunction. In our case, the patient had no hepatic dysfunction throughout the hospital stay; however, we had to delay chest wall stabilization for the patient's safety. Although reoperation was delayed for a month, tissue adhesions were not as severe as expected. In addition, the separated chest wall tissue was well maintained without necrotic changes on follow-up radiography or laboratory findings.

In this case, SSRF was key to weaning the patient from mechanical ventilation and speeding recovery. Although rib fixation surgery, including flail chest stabilization, is still controversial, we suggest that it may provide an option for definitive treatment in appropriately selected patients, even in delayed repair cases. If thoracotomy is required in a patient with flail chest, "on the way out" SSRF may be a feasible option.

\section{CONFLICTS OF INTEREST}

No potential conflict of interest relevant to this article was reported.

\section{INFORMED CONSENT}

This type of study does not require informed consent.

\section{ACKNOWLEDGEMENTS}

This work was supported by clinical research grant from Pusan National University Hospital in 2020.

\section{REFERENCES}

1. Liman ST, Kuzucu A, Tastepe AI, Ulasan GN, Topcu S. Chest injury due to blunt trauma. Eur J Cardiothorac Surg 2003;23:3748.

2. Nirula R, Diaz JJ Jr, Trunkey DD, Mayberry JC. Rib fracture repair: indications, technical issues, and future directions. World J Surg 2009;33:14-22.

3. Todd SR, McNally MM, Holcomb JB, Kozar RA, Kao LS, Gonzalez EA, et al. A multidisciplinary clinical pathway decreases rib fracture-associated infectious morbidity and mortality in highrisk trauma patients. Am J Surg 2006;192:806-11.

4. Tanaka H, Yukioka T, Yamaguti Y, Shimizu S, Goto H, Matsuda $\mathrm{H}$, et al. Surgical stabilization of internal pneumatic stabilization? A prospective randomized study of management of severe flail chest patients. J Trauma 2002;52:727-32.

5. Granetzny A, Abd El-Aal M, Emam E, Shalaby A, Boseila A. Surgical versus conservative treatment of flail chest. Evaluation of the pulmonary status. Interact Cardiovasc Thorac Surg 2005;4:583-7.

6. Marasco SF, Davies AR, Cooper J, Varma D, Bennett V, Nevill R, et al. Prospective randomized controlled trial of operative rib fixation in traumatic flail chest. J Am Coll Surg 2013;216:92432.

7. McDowell A, Dykes J, Paulsen GA. Early reconstruction of the crushed chest. Chest 1962;41:618-23.

8. Moore BP. Operative stabilization of nonpenetrating chest injuries. J Thorac Cardiovasc Surg 1975;70:619-30.

9. París F, Tarazona V, Blasco E, Cantó A, Casillas M, Pastor J, et al. Surgical stabilization of traumatic flail chest. Thorax 1975;30:521-7.

10. Simon B, Ebert J, Bokhari F, Capella J, Emhoff T, Hayward T 3rd, et al. Management of pulmonary contusion and flail chest: an Eastern Association for the Surgery of Trauma practice management guideline. J Trauma Acute Care Surg 2012;73(5 Suppl 4):S351-61.

11. Kors B, Man A, Girbes AR. Conjugated hyperbilirubinaemia in a trauma patient. Netherlands J Critical Care 2009;13:196-9. 